\title{
A Parametrization of Cosmic Ray Shower Profiles Based on Shower Width
}

\author{
J.A.J. Matthews, R. Mesler, B. R. Becker, M. S. Gold, \\ J. D. Hague \\ University of New Mexico, Department of Physics and Astronomy \\ Albuquerque, New Mexico, USA \\ E-mail: johnm@phys.unm.edu
}

\begin{abstract}
Cosmic ray (CR) air showers, detected via the air fluorescence technique, are reconstructed in part using functions that parameterize the longitudinal profile of each shower. The profile parameterization yields the position of shower maximum, $X_{\max }$ in $\mathrm{gm} / \mathrm{cm}^{2}$, which is sensitive to the incident CR particle type: e.g. $\mathrm{p}, \mathrm{C} / \mathrm{N} / \mathrm{O}$, Fe or $\gamma$. The integral of the profile is directly related to the shower energy. The Pierre Auger fluorescence reconstruction uses the Gaisser-Hillas (GH) 4-parameter form[5]. The HiRes group has used both the Gaisser-Hillas form and a 3-parameter Gaussian in Age form[7]. Historically analytic shower theory suggested yet other forms; the best known is a 3-parameter form popularized by Greisen[6]. Our work now uses the shower full width half-maximum, $f w h m$, and shower asymmetry parameter, $f$, to unify the parameterization of all three profile functions. Furthermore shower profiles expressed in terms of the new parameters: $f w h m, f$ have correlations greatly reduced over e.g. Gaisser-Hillas parameters $X_{0}, \lambda$. This allows shower profile reconstructions to add constraints (if needed) on the mostly uncorrelated parameters $f w h m, f$.
\end{abstract}

Keywords: cosmic-ray, air showers, shower profile

PACS: 96.50sd, 96.50.sb

Submitted to: J. Phys. G: Nucl. and Part. Phys.

Dated: 24 November 2009 


\section{Introduction}

Following the first observation of extensive air showers by the air fluorescence technique[1], the Fly's Eye experiment pioneered analysis techniques[2, 3, 4] that are largely unchanged to this day. Most relevant to this paper are the analysis techniques needed to exploit the feature of fluorescence detectors to observe directly the development profile of air showers in the atmosphere. The shower profile provides a direct measurement of the depth of shower maximum, $X_{\max }$ in $\mathrm{gm} / \mathrm{cm}^{2}$, and provides an almost model-independent measurement of the cosmic ray energy[4].

Historically, the shower profile was parameterized[2] using three parameter Gaussian or four parameter Gaisser-Hillas[5] (GH) functions to characterize the shower brightness versus shower depth, $X\left(\mathrm{gm} / \mathrm{cm}^{2}\right)$. The Gaussian parameterization was appealing, as it depended on distance from shower maximum, $X-X_{\max }$, and depended on one parameter to characterize the shower width. As the precision of fluorescence detector measurements improved the Gaussian parameterization was discarded, as it did not model the true shape of shower profiles that are asymmetric in shower depth with respect to shower maximum. The use of the four parameter GH function was often limited by the realities of data signal uncertainties or by the limited fraction of the shower profile observed for any given shower.

More recently the HiRes/MIA collaboration[7] analyzed a compositeł shower profile using three functional forms: Gaisser-Hillas[5] (noted previously), a form motivated by analytic shower theory and popularized by Greisen[6] (Greisen), and a new form motivated by the observation that showers were rather symmetric when plotted in shower age, termed Gaussian in Age (GIA)[7]. Interestingly, the three functions were almost indistinguishable and described the data comparably well. The paper found a strong correlation between two of the GH parameters and suggested that a simpler (3 parameter) form should be adequate to describe the shower profiles. A closely related comparison of GH and GIA functions to Monte Carlo simulated showers[8] generated using CORSIKA[9], again found comparably good agreement using GH or GIA profile functions. Interestingly the Monte Carlo study[8] observed the near equality of the width at half-maximum, fwhm, of proton, iron and photon showers but did not exploit this fact.

Motivated by the history above, in this paper we write the GH, GIA and Greisen functions in terms of 4 parameters: the intensity at shower maximum, $N_{\max }$, the depth of shower maximum, $X_{\max }$, and two other parameters that in the case of $\mathrm{GH}$ are the parameters $X_{0}$ and $\lambda \S$. In all cases the profile functions can then be re-expressed in terms of a physical distance $\Delta=X-X_{\max }$, a composite parameter, $W \equiv X_{\max }-X_{0}$,

$\ddagger$ Showers observed by the HiRes prototype/MIA detectors were aligned to have a common shower maximum. The showers were then averaged to obtain a composite shower profile that was then used in their analysis.

$\S$ While the conventional versions of GIA and Greisen are recovered setting $X_{0}=0$, the introduction of $X_{0}$ allows for a more symmetrical comparison to $\mathrm{GH}$ as well as allowing these forms to be applied to e.g. neutrino induced showers. 
and a (forth) dimensionless parameter, $\xi$. We will now show that $W$ depends both on shower width, e.g. fwhm, and on a shower asymmetry parameter, $f$. The parameter, $\xi$ depends only on the shower asymmetry, $f$.

\section{Profile functions}

We now summarize the three profile functions: GH, GIA and Greisen.

\subsection{Gaisser-Hillas}

The Gaisser-Hillas function[5] is conventionally written:

$$
N(X)_{G H}=N_{\max }\left(\frac{X-X_{0}}{X_{\max }-X_{0}}\right)^{\frac{X_{\max }-X_{0}}{\lambda}} e^{-\frac{X-X_{\max }}{\lambda}}
$$

If we introduce the new variable: $W \equiv X_{\max }-X_{0}$ and the distance from shower maximum: $\Delta=X-X_{\max }$, the $\mathrm{GH}$ function can then be written as:

$$
N(X)_{G H}=N_{\max }\left(1+\frac{\Delta}{W}\right)^{\frac{W}{\lambda}} e^{-\frac{\Delta}{\lambda}}
$$

It is useful to introduce two dimensionless quantities: $\epsilon \equiv \frac{\Delta}{W}$ and $\xi \equiv \frac{W}{\lambda}$. Then a minimal form, useful for comparisons, is obtained:

$$
N\left(X ; N_{\max }, X_{\max }, X_{0}, \lambda\right)_{G H}=N_{\max }(1+\epsilon)^{\xi} e^{-(\epsilon \xi)}
$$

This form emphasizes the role of a physical quantity, the distance from shower maximum, $\Delta$, in comparison to e.g. an unphysical quantity, $X-X_{0}$. Furthermore $\Delta$ is scaled by a parameter $W$ potentially resolving a tension between $X_{\max }$ and $X_{0}$ in a parameter optimization to fit experimental shower profiles.

While the new variables, $W, \xi$, are less intuitive than the standard GH parameters $X_{0}, \lambda$, we will show that they are related to two very intuitive parameters: the shower width, $f w h m$ and a dimensionless shower asymmetry parameter, $f$.

For later reference the integral of the GH function[18] is given by:

$$
\int_{0}^{\infty} N(X)_{G H} d X=N_{\max } W \xi^{-(\xi+1)} e^{\xi} \Gamma(\xi+1)
$$

where $\Gamma$ is the Euler Gamma function.

\subsection{Gaussian in Age}

The Gaussian in Age function[7] is conventionally written:

$$
N(X)_{G I A}=N_{\max } e^{-\frac{1}{2}\left(\frac{s-1}{\sigma}\right)^{2}}
$$

where " $s$ " is the shower age and $s=1$ at shower maximum. Motivated by the definition

of the GH function, the conventional definition for $s_{c o n v}=\frac{3 X}{X+X_{\max }}$ is then modified by replacing: $X \rightarrow X-X_{0}$ and $X_{\max } \rightarrow X_{\max }-X_{0}$. Then with the same definitions as used 
for the GH function (above), the redefined age variable is: $s(\epsilon)=\frac{1+\epsilon}{1+\epsilon / 3}$. Note that this definition preserves $s=1$ at shower maximum and $s=3$ in the limit of $X>X_{\max }$, but differs from "s $s_{\text {conv }}$ " at other shower depths. As with the GH function, a minimal form for the GIA function is obtained:

$$
N\left(X ; N_{\max }, X_{\max }, X_{0}, \sigma\right)_{G I A}=N_{\max } e^{-2\left(\frac{\epsilon}{(3+\epsilon) \sigma}\right)^{2}}
$$

\subsection{Greisen}

The Greisen function[6] is conventionally written[6, 7]:

$$
N(t)_{\text {Greisen }}=N_{\max } e^{\left(t\left(1-\frac{3}{2} \ln (s)\right)-t_{\max }\right)}
$$

where $t$ is the depth in the shower in radiation lengths, $t_{\max }$ is the depth of shower maximum and " $s$ " is shower age. Following the modification of GIA: $t \rightarrow t-t_{0}$ and $t_{\text {max }} \rightarrow t_{\text {max }}-t_{0}$, then converting from depth in radiation lengths to depth in $\mathrm{gm} / \mathrm{cm}^{2}$,

$t=\frac{X}{36.7}$, and using the same notation as for GH and GIA (above) we obtain the minimal form:

$$
N\left(X ; N_{\text {max }}, X_{\text {max }}, X_{0}, p_{36.7}\right)_{\text {Greisen }}=N_{\text {max }} e^{\left(\epsilon\left(1-\frac{3}{2} \ln (s(\epsilon))\right)-\frac{3}{2} \ln (s(\epsilon))\right) \frac{W}{p_{36.7}}}
$$

where the conversion constant " $36.7 \mathrm{gm} / \mathrm{cm}^{2} "$ (per radiation length) is treated as a parameter[7], $p_{36.7} \|$.

It is interesting to note that Eqn. 2, 4 and 5 depend only on $\epsilon \equiv \frac{X-X \max }{W}$ and one additional dimensionless parameter: $\xi \equiv \frac{W}{\lambda}, \sigma$ or $\frac{W}{p_{36.7}}$. As noted above, we will now show that $W$ depends on both shower width and asymmetry: $f w h m, f$ and $\xi$ depends only on shower asymmetry: $f$.

\subsection{Profile parameters: fwhm and $f$}

While there are now some similarities between the three profile functions, a simple connection between the profile parameters and the appearance of a shower does not exist. A natural choice is to use the width of the shower (at e.g. half-height), fwhm and an asymmetry parameter, $f$. Using the profile in Fig. 1 (left), fwhm $\equiv \mathcal{L}+\mathcal{R}$ and $f \equiv \mathcal{L} /(\mathcal{L}+\mathcal{R})$. Thus $f w h m$ has dimensions $\left(\mathrm{gm} / \mathrm{cm}^{2}\right)$ and $f$ is dimensionless. Furthermore, because real and simulated shower profiles rise more steeply than they fall $f$ will always be less than 0.5 .

CORSIKA simulations[8] had suggested that shower fwhm values were rather similar for $\mathrm{p}$, iron and $\gamma$ showers. Results of CONEX $[10,11,12]$ simulated showers are shown in Fig. 2 for $f w h m$ and for the asymmetry parameter, $f$, for shower energies relevant to the Auger[13], HiRes[14] and TA[15] experiments. These simulations are consistent with the CORSIKA results and suggest that for energies $\sim 10^{18.5} \mathrm{eV}$ and for all primaries the fwhm is in the rather limited range: $475 \mathrm{gm} / \mathrm{cm}^{2} \lesssim f w h m \lesssim 575$ $\mathrm{gm} / \mathrm{cm}^{2}$ and asymmetry in the range: $0.44 \lesssim f \lesssim 0.47$. Furthermore the CONEX

\| The original Greisen functional form is simply regained by setting: $X_{0}=0$ and $p_{36.7}=36.7$. 

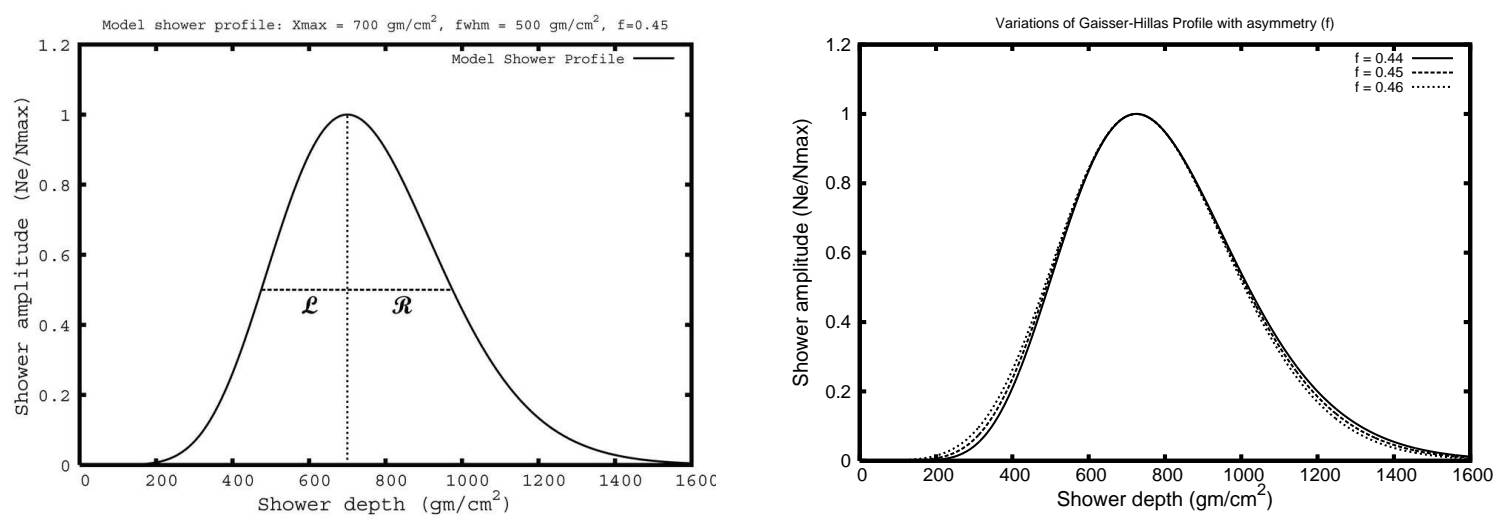

Figure 1. Left: Plot of a representative Gaisser Hillas (GH) shower profile defined by: $X_{\max }=700 \mathrm{gm} / \mathrm{cm}^{2}, f w h m \equiv \mathcal{L}+\mathcal{R}=500 \mathrm{gm} / \mathrm{cm}^{2}$ and shower asymmetry $f \equiv \mathcal{L} /(\mathcal{L}+\mathcal{R})=0.45$. The vertical axis is the shower intensity $\left(N_{e}\right)$ normalized to the intensity at shower maximum $\left(N_{\max }\right)$ and the horizontal axis is the shower depth $(X)$ in the atmosphere in $\mathrm{gm} / \mathrm{cm}^{2}$. Right: GH shower profiles with $X_{\max }=725$ $\mathrm{gm} / \mathrm{cm}^{2}, f w h m=525 \mathrm{gm} / \mathrm{cm}^{2}$ and three different values of asymmetry: $f=0.44$, 0.45 and 0.46. The corresponding GH parameters are: $\left(X_{0}=2.2 \mathrm{gm} / \mathrm{cm}^{2}, \lambda=68.3\right.$ $\left.\mathrm{gm} / \mathrm{cm}^{2}\right),\left(X_{0}=-145 \mathrm{gm} / \mathrm{cm}^{2}, \lambda=56.9 \mathrm{gm} / \mathrm{cm}^{2}\right)$ and $\left(X_{0}=-365 \mathrm{gm} / \mathrm{cm}^{2}, \lambda=45.5\right.$ $\left.\mathrm{gm} / \mathrm{cm}^{2}\right)$ respectively.

simulations suggest that the asymmetry parameter, $f$, may provide some discrimination in primary composition[16, 17], i.e. between different types of primary particles. Finally both fwhm and asymmetry, $f$, show a slow, approximately logarithmic increase with increasing shower energy.
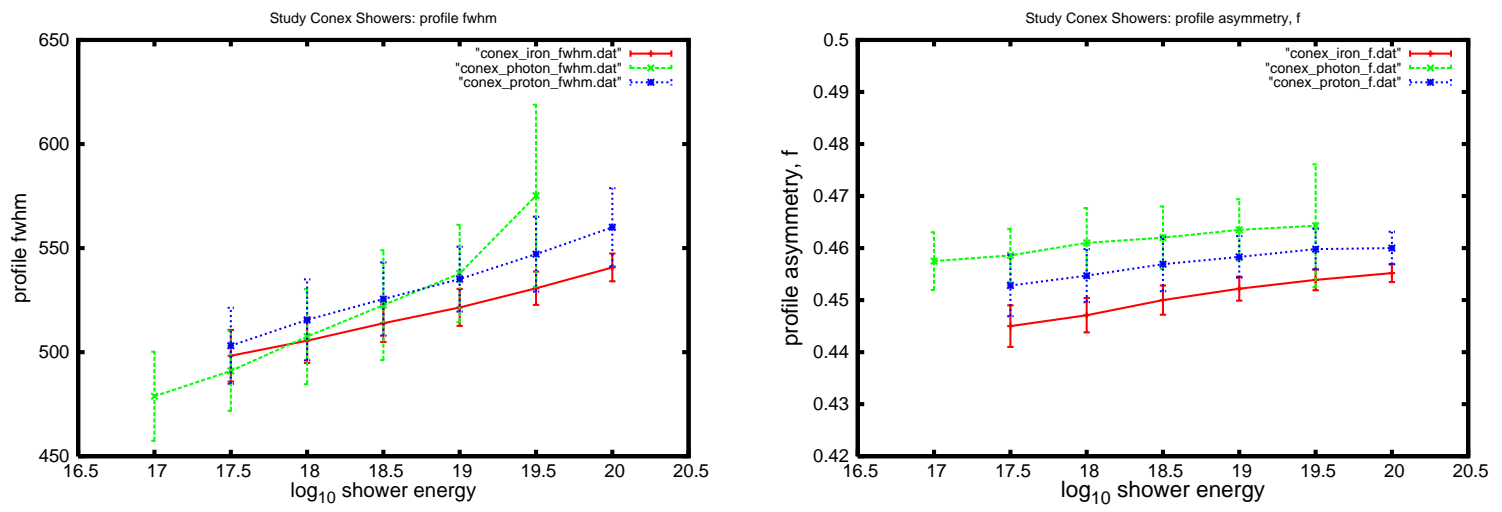

Figure 2. The results of CONEX[10] simulations of proton, iron and $\gamma$ initiated air showers are shown in blue, red and green respectively. Left: Plot showing the shower widths $(f w h m)$ on the vertical axis versus the shower energies on the horizontal axis. The error bars give the RMS of the simulated showers in each energy bin. Right: Plot showing the shower asymmetries $(f)$ on the vertical axis versus the shower energies on the horizontal axis. 


\subsection{Relating old and new shower parameters}

To relate the old to the new shower parameters, the shower profile is evaluated at a fraction $h$ of maximum. $N(X)=h N_{\max }$. To illustrate the procedure we choose the GH profile as written in Eqn. 1. Then solving for the values of $\Delta$ that satisfy $N(X)=h N_{\max }$ one obtains:

$$
\frac{W}{\lambda} \ln \left(1+\frac{\Delta}{W}\right)-\frac{\Delta}{\lambda}=\ln (h)
$$

This relation is true for two values of $\Delta$, see Fig. 1(left): $\Delta_{\mathcal{L}}=(-f(h))$ Width $(h)$ and $\Delta_{\mathcal{R}}=(1-f(h))$ Width $(h)$ where $\left|\Delta_{\mathcal{L}}\right|+\Delta_{\mathcal{R}}=W i d t h(h)$ is the width of the shower profile curve at fractional height $h \boldsymbol{q}$. Following the familiar convention to describe a profile at half-height, we define $f w h m \equiv W i d t h(h=0.5)$ and $f \equiv f(h=0.5)$.
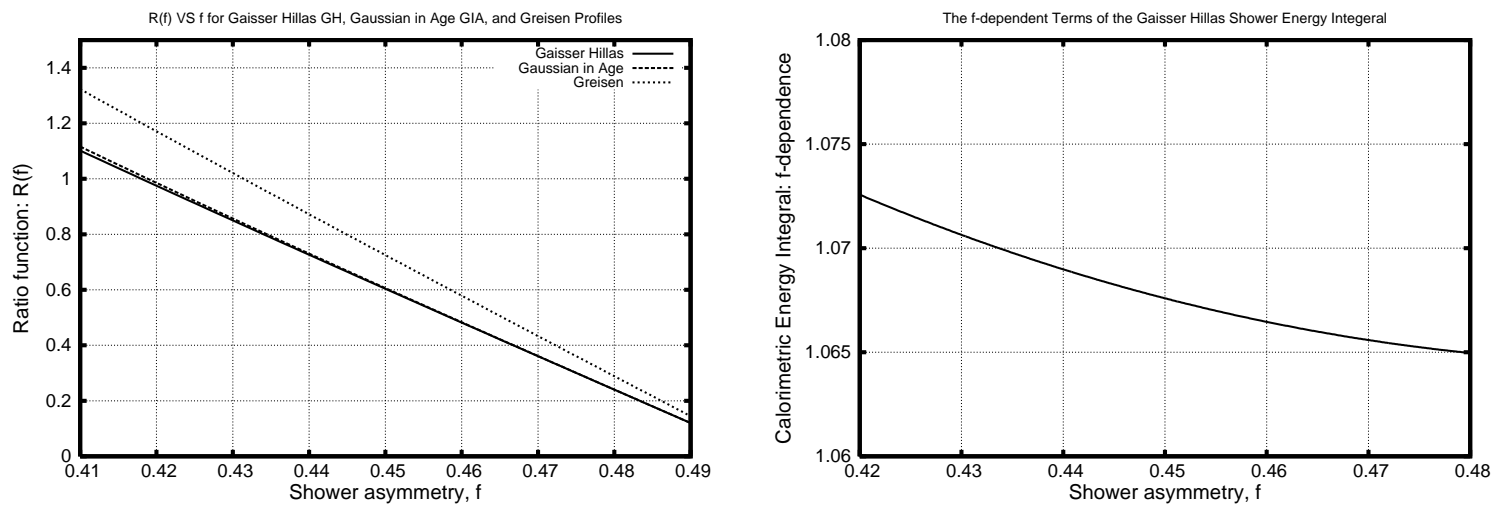

Figure 3. Left: The curves show the ratio function, $R(f)$, plotted on the vertical axis versus the shower asymmetry, $f$, on the horizontal axis. As discussed in the text the ratio function: $R \equiv \frac{f w h m}{W}=\frac{f w h m}{X_{\max }-X_{0}}$ depends only on the shower asymmetry. The ratio functions for the different shower profiles are plotted as: GH (solid), GIA (dashes) and Greisen (dots). Right: The asymmetry $f$-dependent terms in Eqn. 7, $\frac{\xi^{-(\xi+1)} e^{\xi} \Gamma(\xi+1)}{R(f)}$, are plotted on the vertical axis versus the shower asymmetry, $f$, on the horizontal axis.

The equations for $\Delta_{\mathcal{L}}$ and $\Delta_{\mathcal{R}}$ can be combined to obtain one (GH profile specific) relationship:

$$
\ln \left(\frac{1-f R}{1+(1-f) R}\right)+R=0
$$

where $R \equiv \frac{f w h m}{W}$ depends only on the shower asymmetry, $f$. Eqn. 6 is solved numerically to obtain the ratio function: $R=R(f)$; the result is shown in Fig. 3(left).

The solution to Eqn. 6 relates $W$ to the shower $f w h m: W=\frac{f w h m}{R(f)}$. Finally with the ratio function $R(f)$ and $W$ known, the GH parameters $X_{0}$ and $\lambda$ are given by:

I While both Width $(h)$ and $f(h)$ depend on the choice for $h$, the shower shape and the parameters describing the shower shape, e.g. GH parameters $X_{0}, \lambda$, do not. 


$$
\begin{gathered}
X_{0}=X_{\max }-W \\
\lambda=\frac{W[\ln (1-f R)+f R]}{\ln (h=0.5)}
\end{gathered}
$$

It is important to note that $\xi=\frac{W}{\lambda}$ depends only on the asymmetry parameter, $f$. Thus the calorimetric shower energy, given by:

$$
E_{\text {shower }}^{\text {calor }}=\int_{0}^{\infty} N(X)_{G H} d E / d x d X
$$

is to a good approximation[19], see Eqn. 3:

$$
E_{\text {shower }}^{\text {calor }}=\left\langle d E / d x>N_{\max } f w h m\left(\frac{\xi^{-(\xi+1)} e^{\xi} \Gamma(\xi+1)}{R(f)}\right)\right.
$$

where $\left\langle d E / d x>\approx 2.19 \mathrm{MeV} /\right.$ particle $/\left(\mathrm{gm} / \mathrm{cm}^{2}\right)[19]$ and $N_{\max }$ is the number of charged particles at shower maximum. The dependence on shower asymmetry, $f$ is shown in Fig. 3(right) and is $\sim 1 \%$ over the range of asymmetries predicted using CONEX, see Fig. 2(right). Thus to a good approximation the shower calorimetric energy is proportional to: $N_{\max } f w h m$; see Fig. 4 . This relation emphasizes the importance of the shower width, fwhm, as errors in determining the width directly propagate into errors in estimating the shower energy. This relation also shows that mis-measuring the asymmetry has almost no effect on the shower energy. Eqn. 7 and Fig. 3(right) do imply a small correlation coming from the calorimetric shower energy constraint: namely that more asymmetric showers (smaller values of $f$ ) should have slightly smaller values of fwhm.

To relate conventional GIA parameters, $X_{0}, \sigma$, to new parameters, $f w h m, f$, we follow the same procedure that was employed for the GH function. For the GIA profile, the simple mathematical form of the GIA function (see Eqn. 4) results in an analytic (GIA profile specific) solution for the ratio function $R(f)$ :

$$
R(f)=\frac{3(1-2 f)}{2 f(1-f)}
$$

which is plotted in Fig. 3(left). As with GH, $W=\frac{f w h m}{R(f)}$. The conventional GIA parameters are then given by:

$$
\begin{gathered}
X_{0}=X_{\max }-W \\
\sigma=\frac{f R}{(3-f R) \sqrt{\frac{-\ln (h=0,5)}{2}}}
\end{gathered}
$$

Finally to relate conventional Greisen parameters, $X_{0}, p_{36.7}$, to new parameters, $f w h m, f$, the first step is to solve for the values of $\Delta$ that satisfy $N(X)=h N_{\text {max }}$ : 


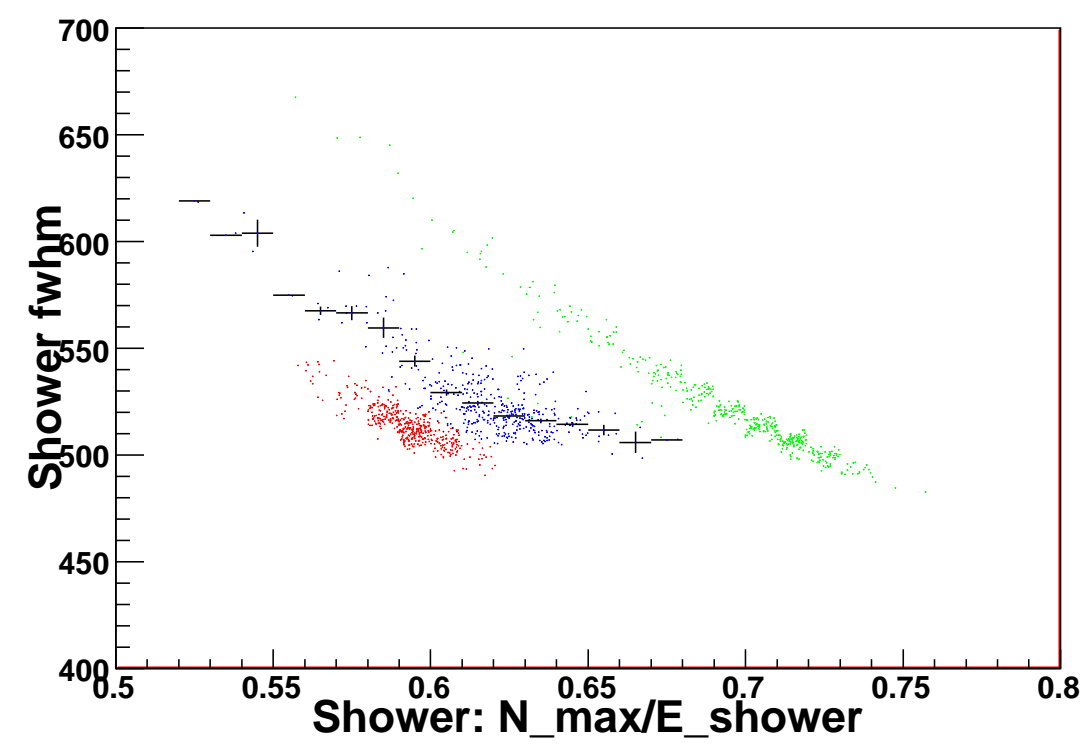

Figure 4. Scatter plot showing CONEX simulations of proton, iron and $\gamma$ initiated air showers shown in blue, red and green respectively. The points report the shower $f w h m$ in $\left(\mathrm{gm} / \mathrm{cm}^{2}\right)$ on the vertical axis versus the ratio of shower quantities: $N_{\max } / E_{\text {shower }}$ in (particles $/ \mathrm{GeV}$ ) on the horizontal axis. See Eqn. 7 for motivation. All showers had an energy near $10^{18.5} \mathrm{eV}$. The relative (horizontal) displacement of the iron $: \mathrm{p}: \gamma$ showers is related to increasing missing energy (in muons and neutrinos) in iron versus $\mathrm{p}$ versus $\gamma$ showers. The points with error-bars reflect the average of the proton points in the scatter plot.

$$
\frac{\Delta}{W}\left(1-\frac{3}{2} \ln (s)\right)-\frac{3}{2} \ln (s)=\frac{p_{36.7}}{W} \ln (h)
$$

As with the GH function, this relation is true for two values of $\Delta_{1} \Delta_{\mathcal{L}}$ and $\Delta_{\mathcal{R}}$. The two resulting equations can be combined into one relationship that relates $R \equiv \frac{f w h m}{W}$ to the shower asymmetry, $f$. The solution, found numerically, is plotted in Fig. 3(left). Once the ratio function $R(f)$ is determined, then the Greisen parameters, $X_{0}, p_{36.7}$ are given by:

$$
\begin{gathered}
X_{0}=X_{\max }-W \\
p_{36.7}=\frac{W}{\ln (h=0.5)}\left[-f R\left(1-\frac{3}{2} \ln \left(s_{\mathcal{L}}\right)\right)-\frac{3}{2} \ln \left(s_{\mathcal{L}}\right)\right]
\end{gathered}
$$

where $s_{\mathcal{L}} \equiv s\left(\frac{\Delta_{\mathcal{L}}}{W}\right)$ is the (redefined) shower age (see Sect. 2.2 above).

\section{Profile comparisons}

Now that the GH, GIA and Greisen functions can be expressed in common parameters: $f w h m, f$, it is easy and instructive to compare their shower profiles. An example 

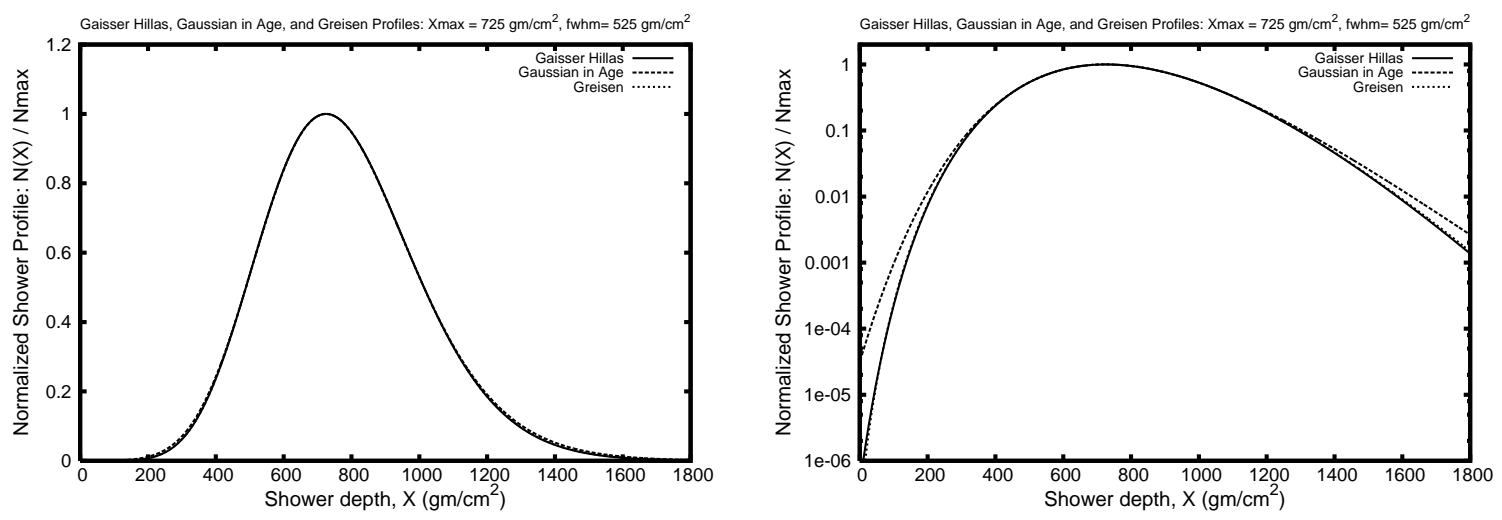

Figure 5. Left: Plot of shower profiles for GH (solid), GIA (dashes) and Greisen (dots) profile functions for typical shower profile parameters: $X_{\max }=725 \mathrm{gm} / \mathrm{cm}^{2}$, $f w h m=525 \mathrm{gm} / \mathrm{cm}^{2}$, and $f=0.45$. The vertical axis is the shower intensity normalized to the intensity at shower maximum and the horizontal axis is the shower depth in the atmosphere in $\mathrm{gm} / \mathrm{cm}^{2}$. Right: Same shower profiles now with a semi-log plot to show the small differences between the functions.

comparison, shown in Fig. 5, finds that GH and Greisen functions are almost indistinguishable while (both) being very similar to, but systematically below the GIA profile for shower depths well away from shower maximum. This result is consistent with HiRes/MIA[7] and HiRes[8] observations that the profiles describe experimental (and simulated) shower data comparably well near shower maximum. Quantitatively, the small but systematic profile function differences result in shower calorimetric energies evaluated using the GIA function being $~ 1 \%$ larger than those evaluated using GH or Greisen forms.

As noted in Sect 2.4, the shower profiles away from shower maximum may provide some discrimination on cosmic ray composition[16, 17] (cf. Fig. 2(right)) and on whether the GIA[20] versus GH (or Greisen) profiles provide somewhat better parameterizations of actual shower profiles. While interesting, this is not the thrust of this paper and will not be discussed further.

A remaining issue is how best to apply four parameter shower profile functions to less than ideal data. In these circumstances the tendency is to fix, or constrain, one or more of the parameters. An equivalent point of view is the assumption that $X_{0}=0$ $\mathrm{gm} / \mathrm{cm}^{2}$ in the GIA or Greisen function and/or that the parameter $p_{36.7}=36.7 \mathrm{gm} / \mathrm{cm}^{2}$ (per radiation length) in the Greisen function.

To study this issue, shower profile parameters from CONEX showers are shown in Fig. 6 for proton showers with energies $\sim 10^{18.5} \mathrm{eV}$. For each simulated shower, the shower fwhm and asymmetry, $f$, are determined. The values are plotted in Fig. 6(lower right). Following Sect. 2.5 the fwhm and $f$ values are used to determine the corresponding parameters for GH, GIA and Greisen profiles. The results are plotted in the other quadrants of Fig. 6. The conventional shower shape parameters: $\left(X_{0}, \lambda\right),\left(X_{0}, \sigma\right)$, and $\left(X_{0}, p_{36.7}\right)$ show significant correlations while $(f w h m, f)$ do not. In particular 

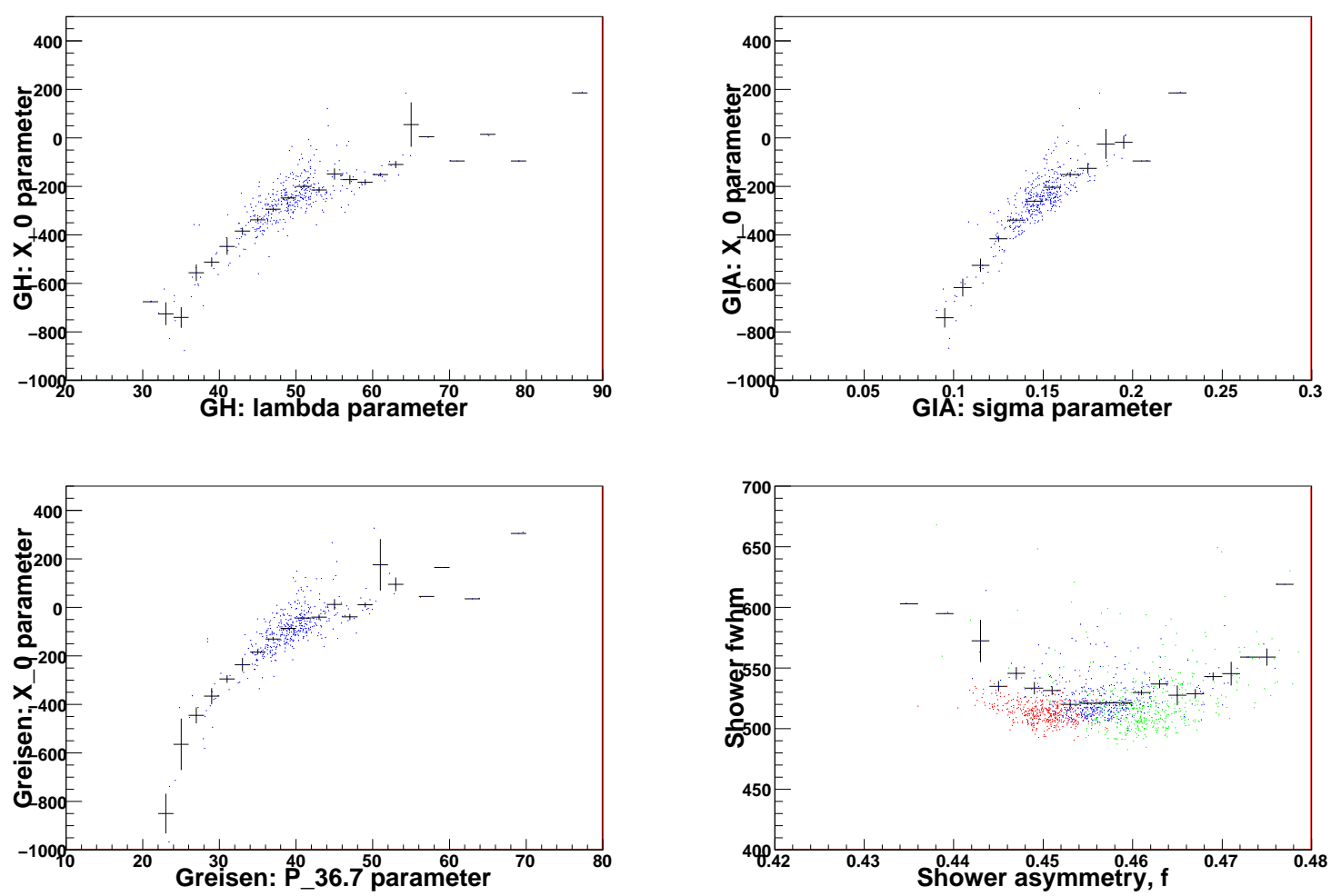

Figure 6. Scatter plots of Top Left: GH parameters $\left(X_{0}, \lambda\right)$, Top Right: GIA parameters $\left(X_{0}, \sigma\right)$, Bottom Left: Greisen parameters $\left(X_{0}, p_{36.7}\right)$ for proton and Bottom Right: shower $(f w h m, f)$ for proton (blue), iron (red) and $\gamma$ (green) showers near $10^{18.5} \mathrm{eV}$ simulated with CONEX. In all cases the first parameter is plotted on the vertical axis and the second parameter on the horizontal axis. The points with errorbars reflect the average of the (proton) points in the scatter plot. The plot statistics are: 411 proton, 440 iron and 462 photon CONEX showers.

the correlation coefficients for GH, GIA and Greisen parameters are in the range: $0.76 \sim 0.91$ for CONEX[10, 11, 12] proton and iron showers while the correlation coefficients for $(f w h m, f)$ are $\sim 0.12$ for proton showers and $\sim-0.11$ for iron showers.

While the GH correlation results appear consistent with the observation of parameter correlations in Ref.[7], our analysis does not (then) support the use of GIA (or Greisen) shower profiles with the $X_{0}$ parameter set to zero[7]. Rather than the correlation being evidence that three parameters are sufficient to describe shower profiles, it is more likely that GH, GIA and Greisen parameters need to fulfill the calorimetric energy constraint of Eqn. $7^{+}$. As noted above, this constraint is almost insignificant with the choice of variables $(f w h m, f)$. In summary the $(f w h m, f)$ shower shape parameters appear less correlated that the other shower parameter pairs (while still assuming a wide range of values).

That said, it is likely that shower details lead to correlations with any choice of

+ An almost equivalent statement, is that e.g. $\left(X_{0}, \lambda\right)$ must be compatible with the full with half maximum of the shower. 
shower parameters. In that regard, this study is compatible with shower reconstructions using partial constraints[18]; we recommend that the constraints be applied to $f w h m$ and $f$ rather than the $\mathrm{GH}$ parameters $X_{0}$ and $\lambda$.

\section{Conclusion}

This analysis has studied three functions used to parameterize the shower profiles of ultra-high energy cosmic rays: Gaisser-Hillas (GH), Gaussian in Age (GIA) and an analytical form popularized by Greisen (Greisen). As part of this analysis, we have generalized the GIA and Greisen functions to 4-parameters consistent with the GH function. To then study the relation between the three shower parameterizations, and to use more physically descriptive parameters, this work uses the shower full width halfmaximum, fwhm, and shower asymmetry parameter, $f$, to parameterize the shape of the shower profiles. For profiles with the same $(f w h m, f)$, the GH and Greisen shower profiles are essentially identical and systemically less than GIA for shower depths away from shower maximum. Of the three functions, $\mathrm{GH}$ is most convenient as the integral of the GH profile is an analytic function. Monte Carlo simulated air showers using CONEX, and parameterized in terms of the new parameters: ( $f w h m, f)$, have correlations (between those parameters) greatly reduced over the standard parameterizations e.g. Gaisser-Hillas parameters: $\left(X_{0}, \lambda\right)$. This allows shower profile reconstructions to add constraints (if needed) on the mostly uncorrelated parameters $f w h m, f$. The CONEX shower simulations suggest that the shower asymmetry parameter, $f$, may have some sensitivity to the incident cosmic ray particle type: e.g. p, C/N/O, Fe or $\gamma$.

\section{Acknowledgments}

We wish to recognize discussions with Miguel Mostafa, Paul Sommers, Jaroslav Urbar and Marcelo Vogel during early work on this paper. We also recognize and appreciate support by DOE grant DE-FR02-04ER41300.

\section{References}

[1] H.E. Bergeson et al, Measurement of Light Emission from Remote Cosmic-ray Showers. Phys. Rev. Lett. 39847 (1977)

[2] R.M. Baltrusaitis et al, The Utah Fly's Eye Detector. Nucl. Instr. Meth. A240 410 (1985)

[3] G.L. Cassiday et al, Measurement of Cosmic-ray Air Shower Development at Energies above $10^{17} \mathrm{eV}$. Astrophysical J. 356669 (1990)

[4] D.J. Bird et al, The Cosmic-ray Energy Spectrum Observed by the Fly's Eye. Astrophysical J. 424491 (1994)

[5] T.K. Gaisser and A.M. Hillas, Reliability of the Method of Constant Intensity Cuts for Reconstructing the Average Development of Vertical Showers. Proc. 15th ICRC 8353 (1977)

[6] K. Greisen, The Extensive Air Showers. Prog. in Cosmic Ray Physics, III, 1 (1956)

[7] T. Abu-Zayyad et. al, A Measurement of the Average Longitudinal Development Profile of Cosmicray Showers between $10^{17}$ and $10^{18} \mathrm{eV}$. Astropart. Phys 161 (2001) astro-ph/0008206 
[8] C. Song et al, Study of the Longitudinal Development of Air Showers with CORSIKA. Proc. of ICRC 2001, 490 (2001)

[9] D. Heck et al, CORSIKA: A Monte Carlo Code to Simulate Extensive Air Showers. Report FZKA 6019 Forschungszentrum Karlsruhe (1998)

[10] T. Pierog et al., First Results of Fast One-dimensional Hybrid Simulation of EAS Using CONEX. Nucl. Phys. Proc. Suppl. 151159 (2006) astro-ph/0411260

[11] N.N. Kalmykov, S.S. Ostapchenko and A.I. Pavlov, Nucl. Phys. Proc. Suppl. 52B 17 (1997)

[12] R. Engel et al., Proc. 26th ICRC, 1415 (1999)

[13] J. Abraham et al, The Fluorescence Detector of the Pierre Auger Observatory. arXiv:0907.4282 (2009)

[14] T. Abu-Zayyad et al., Status of the High Resolution Fly's Eye Detector: Operation and Installation. Proc. 26th ICRC, 5349 (1999); J.H. Boyer et al., Nucl. Inst. Meth. A482 457 (2002)

[15] H. Kawai et al., Telescope Array: Status and Prospects. Proc. 29th ICRC, 8141 (2005)

[16] S. Andringa, R. Conceicao, M. Pimenta, Increased Sensitivity to Electromagnetic and Hadronic Features of Air Showers from a New Parameterization of the Longitudinal Profiles. Proc. 31st ICRC, Lodz, Poland (2009)

[17] V. Scherini, F. Schussler, R. Engel, K.-H. Kampert, M. Risse, M. Unger, Simulation Study of Shower Profiles from UHE Cosmic Rays. Proc. 30th ICRC, Merida, Mexico (2007)

[18] M. Unger et al, Reconstruction of Longitudinal Profiles of UHE Cosmic-ray Showers from Fluorescence and Cherenkov Light Measurements. Nucl. Instr. Meth. A588 433 (2008) arXiv:0801.4309v1.

[19] C. Song et al, Energy Estimation of UHE Cosmic Rays using the Atmospheric Fluorescence Technique. Astropart. Phys. 147 (2000)

[20] M. Giller, A. Kacperczyk, J. Malinowski, W. Tkaczyk, G. Wieczorek, Similarity of Extensive Air Showers with Respect to Shower Age. J. Phys. G: Nucl. Part. Phys. 31947 (2005) 